\title{
Intraoperative Portal Vein Thrombosis after Pancreatic and Partial Portal Vein Resections
}

\author{
Gordillo $\mathrm{A}^{1 *}$, Nogales $\mathrm{A}^{1}$, Araji $\mathrm{O}^{2}$, Sanchez-Matamoros $\mathrm{I}^{1}$, Perez $\mathrm{A}^{1}$ and Oliva $\mathrm{F}^{3}$
}

${ }^{1}$ General and Digestive Surgery Unit of Hepatobiliopancreatic, Hospital Universitario Virgen Macarena, Spain

${ }^{2}$ Department of Cardiovascular Surgery, Hospital Universitario Virgen Macarena, Spain

${ }^{3}$ Chief of General Surgery Service, Hospital Universitario Virgen Macarena, Spain

\begin{abstract}
We report a case of intraoperative portal vein thrombosis in a patient with pancreatic head adenocarcinoma and tumor infiltration of the anterior wall of portal vein. Advances in surgery allow reparation of these vessels that can be infiltrated by the tumor. Currently we have a wide technical and prosthetic arsenal for this purpose. This case suggests that the use of patches is not a good choice and that the end to end suture is probably the most recommended in cases that require the portal reparation.
\end{abstract}

Keywords: Portal thrombosis; Pericardium patch; Tumour

\section{Introduction}

Advanced gastrointestinal tumors are aggressive and often invade blood vessels. Advances in surgical techniques allow their resection even in presence of vessel infiltration. In cases of portal vein involvement, it is not clear the way it should be reconstructed, some series report reconstruction with patch and few cases are reported for acute intraoperative portal trombosis, because of the patch employment. Here we report a case of such complication.

\section{Presentation of Case}

We report a 58-year-old patient with a history of hypertension, dyslipidemia, ovarian cystectomy and appendectomy that began with right upper quadrant pain and obstructive jaundice. The diagnostic was pancreatic head adenocarcinoma. Contrast Computed Tomography (CT) is performed where nodular hypo intense image is displayed on the rear margin of $2 \mathrm{~cm}$ succinate and is in intimate contact with the rear margin of the portal vein producing deformity thereof remaining permeable. Echo endoscopy - Fine needle aspiration (FNA) where diagnosis is confirmed. A $10 \mathrm{~F}$ plastic stent is placed $5 \mathrm{~cm}+$ suprapapillary sphincterotomy. Subsequently she was admitted for elective surgery.

Bilateral subcostal incision is made. Intraoperative ultrasound without appreciating extension that contraindicates surgery, showing imprint / infiltration of the portal vein by the tumor. Dissection of the hepatic hilum, pyloric and gastro duodenal vessels, lymphadenectomy of the celiac trunk, Kocher maneuver and interaortocaval lymphadenectomy. Antral, pancreatic neck and $1^{\text {st }}$ jejunal loop section. After ligation of Henle confluent, tumor infiltration of the anterior wall of portal vein is seen, resection of that portion with safety margins, performing repair with bovine pericardium, with previous heparinization with 5000 UI. The liver-jejunostomy, gastrojejunostomy transmesocolic handle and Y Roux are continuous with the pancreatic-jejunostomy with end to end anastomosis. We reviewed portal vein malfunction and observed formation of a thrombus proximal to the repair, so we decided heparinize with 5000 UI sodium heparin and remove the patch. Once stuffed the walls, portal, superior mesenteric and splenic proceeds to repair by transverse suture (Figures 1 and 2).

Postoperative short and long term has been favorable and uncomplicated. Low molecular weight heparin administered at prophylactic doses from the first postoperative day. The patient was discharged with good result and is currently on track for oncology.

\section{Discussion}

Fukuda [1], noted that the pancreaticoduodedenectomy with resection of the portal vein may be only potential cure for patients with pancreatic duct adenocarcinoma and involvement of the portal vein. In our case, the portal vein was repaired with a pericardial patch. We present this case to show thrombosis as complication after intraoperative use of pericardial patch to close the defect secondary to vascular invasion by the tumor.

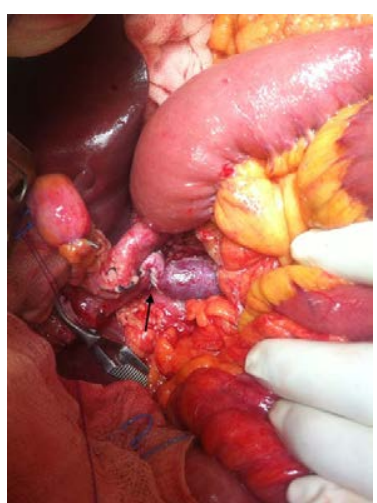

Figure 1: Dissection of the hepatic hilum, pyloric and gastro duodenal vessels, lymphadenectomy of the celiac trunk, Kocher maneuver and interaortocaval lymphadenectomy.

*Corresponding author: Alejandra Gordillo Hernandez, Hospital Universitario Virgen Macarena, Avenida Doctor Fedriani 3, 41071 Sevilla, Spain, Tel: 34-658154-289; Fax: 34-954-557-347; E-mail: alejandragordillo@hotmail.com

Received November 27, 2015; Accepted December 22, 2015; Published January 03, 2016

Citation: Gordillo A, Nogales A, Araji O, Sanchez-Matamoros I, Perez A, et al (2016) Intraoperative Portal Vein Thrombosis after Pancreatic and Partial Portal Vein Resections. J Vasc Med Surg 4: 244. doi:10.4172/2329-6925.1000244

Copyright: $\odot 2016$ Hernandez AG, et al. This is an open-access article distributed under the terms of the Creative Commons Attribution License, which permits unrestricted use, distribution, and reproduction in any medium, provided the original author and source are credited. 
Citation: Gordillo A, Nogales A, Araji O, Sanchez-Matamoros I, Perez A, et al. (2016) Intraoperative Portal Vein Thrombosis after Pancreatic and Partial Portal Vein Resections. J Vasc Med Surg 4: 244. doi:10.4172/2329-6925.1000244

Page 2 of 2

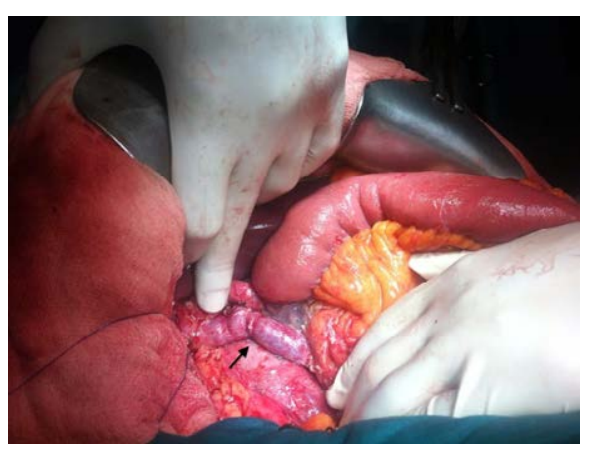

Figure 2: Walls repaired by transverse sutures.

Yekebas [2], published the long-term survival perioperative results of 136 patients who underwent vascular resection for locally advanced pancreatic neoplasia. In 8 cases they had to resect more than one third of the lateral wall and the patches they used were from autologous venous (internal jugular, saphenous, inferior mesenteric) to prevent stenosis. In all patients prophylactic heparinization with low molecular weight is used. They recorded the portal vein thrombosis in 2 of venous resections.

Ravikumar[3],presentastudycomparingpancreaticoduodenectomy with resection of the portal vein or superior mesenteric, standard pancreaticoduodenectomy, and surgical bypass. Seven patients had portal vein thrombosis. Six patients had end to end anastomosis and one with graft interposition. Höing [4], have recently published a case report where they use a Polytetrafluoroethylene (PTFE) graft in endto-end technique.

In our case we used a bovine patch, the portal cossclamp time was as short as 10 minutes, and at the end of th surgery we observed portal vein trombosis, we hypothize that it has occured as a result of a combination of on one hand the prothrombotic status of the patient, and on the other the very low pressure and slow run off of the portal vein that may be prone for thrombosis if we use pericardial patches.
So we believe that it is important to reconstruct the vein whenever is possible directly without any synthetic material.

\section{Conclusion}

Intraoperative thrombosis in this case may be due to slowness, low blood pressure and hypercoagulability factors. So we thought that the use of patches is not a good choice due to the lower pressure of the circulatory system at this level.

We conclude that the end to end suture is probably the most recommended in cases requiring portal resection to repair and intraoperative regimen heparinization with sodium heparin $5000 \mathrm{UI}$ followed by postoperative heparinization.

\section{Conflicts of Interest}

The authors report no conflict of interest.

\section{Funding}

The authors have no funding to declare.

\section{Consent}

Written informed consent was obtained from the patient for publication of this case report and accompanying images.

\section{References}

1. Fukuda S, Oussoultzoglou E, Bachellier P, Rosso E, Nakano H, et al. (2007) Significance of the depth of portal vein wall invasion after curative resection for pancreatic adenocarcinoma. Arch Surg 142: 172-179.

2. Yekebas EF, Bogoevski D, Cataldegirmen G, Kunze C, Marx A, et al. (2008) En Bloc Vascular Resection for Locally Advanced Pancreatic Malignancies Infiltrating Major Blood Vessels Perioperative Outcome and Long-term Survival in 136 Patients. Ann Surg 247: 300-309.

3. Ravikumar R, Sabin C, Hilal MA, Bramhall S, White S, et al. (2013) Portal Vein Resection in Borderline Resectable Pancreatic Cancer: A United Kingdom Multicenter Study. J Am Coll Surg 218: 401-411.

4. Höing K, Ringe KI, Bektas H, Klempnauer J, Jäger MD (2015) Preduodenal superior mesenteric vein and Whipple procedure with vascular reconstruction A case report. Int J Surg Case Rep 10: 107-110. 